\section{Retrospective study of post-operative regurgitation in dogs following upper airway surgery to treat brachycephalic obstructive airway surgery: 150 cases (2013-2016)}

\section{Joy Fenner, Robert Quinn, Jackie Demetriou}

Dick White Referrals, Newmarket, UK

\section{OBJECTIVE}

To determine the incidence of regurgitation in dogs within 24 hours of surgery to treat brachycephalic obstructive airway syndrome (BOAS), including either a staphylectomy or folded flap palatoplasty, and to identify possible risk factors for this complication.

\section{METHOD}

Retrospective single centre study of dogs undergoing BOAS surgery over three years. Electronic medical records were searched for dogs that had undergone a folded flap palatoplasty or staphylectomy as part of BOAS surgery at a UK specialist referral centre. Data retrieved included signalment, surgical procedures performed, grade of laryngeal collapse, history of pre-operative regurgitation, drugs administered, regurgitation whilst hospitalised pre- and post-operatively, length of hospitalisation, and requirement for tracheotomy. These were assessed using univariable binomial logistic regression; results with $\mathrm{P}<0.05$ were then assessed against covariates thus identifying confounding factors in a multivariate model.

\section{RESULTS}

Of 150 dogs meeting the inclusion criteria 46 regurgitated during the first 24 hours postoperatively (30.67\%). Potential risk factors identified from univariate analysis included age, perioperative use of omeprazole, alar cartilage resection as part of surgery, being unneutered and breed (French Bulldog). Following assessment with covariates, age (as a continuous variable) remained significant with younger dogs more at risk. Age ranged from 3 months to 11 years (mean $=3$ years). Of 72 dogs aged 2 or under $41.7 \%(30 / 72)$ regurgitated compared with $20.5 \%$ of dogs aged 2 or older ( $16 / 78$ dogs).

\section{IMPACT}

Dogs of a younger age presenting for BOAS surgery are more likely to regurgitate within 24 hours of surgery than older dogs.

\section{Surgical management of impalement injuries to the trunk of dogs: a multi-centre retrospective study}

\section{Matt Matiasovic ${ }^{1,2}$, Zoe Halfacree ${ }^{3}$, Alison Moores ${ }^{4}$, Pieter Nelissen ${ }^{1}$, Samantha Woods ${ }^{5}$, Barnaby Dean ${ }^{6,2}$, Guillaume Chanoit ${ }^{2}$, Darren Barnes ${ }^{1}$}

\footnotetext{
Dick White Referrals, Newmarket, UK

University of Bristol Small Animal Hospital, Langford, UK Royal Veterinary College, Hatfield, UK

Anderson Moores Veterinary Specialists, Hursley, UK Royal (Dick) School of Veterinary Studies, Edinburgh, UK 6 Southern Counties Veterinary Specialists, Ringwood, UK
}

\section{OBJECTIVES}

The purpose of this retrospective study was to review a large series of dogs that were referred for specialist treatment having suffered traumatic impalement injuries to the thorax and/or abdomen and to report the aetiologies, injury characteristics, surgical management and long-term outcomes for these patients.

\section{METHODS}

Retrospectively collected data of dogs surgically treated for impalement injuries to the trunk at six veterinary referral institutions in the UK over an 11-year period were reviewed. Data collected for each case included patient signalment, physiologic variables, injury-specific variables, diagnostic imaging reports, surgical procedurerelated variables, duration of hospitalisation, antibiotic use, complications and outcomes. All data were reported with summary statistics.

\section{RESULTS}

Fifty-four dogs were included. Impalement occurred most frequently on wooden objects $(n=34)$ and the 


\section{Oral presentations}

thoracic cavity was most commonly penetrated $(n=35)$. Diagnostic imaging (all modalities combined) was sensitive and specific to identifying wooden material in $56 \%$ and $95 \%$ of cases respectively and surgical retrieval of a foreign body or its fragments was required in 20 cases (37\%). Thoracotomy was performed in $56 \%$ of the cases. Complications occurred in 20 dogs $(37 \%)$ and of these $70 \%$ were minor, $10 \%$ major and $20 \%$ catastrophic.

\section{STATEMENT}

Despite the often seen dramatic presentation of impalement injuries the majority of treated patients can achieve good outcomes. These injuries require thorough diagnostic imaging and interpretation prior to adequate surgical exploration and management, augmented by anaesthetic and critical care during the peri- and postoperative periods; therefore stable patients should be referred to centres able to provide this type of care.
Retrospective evaluation of surgical treatment for congenital extrahepatic portosystemic shunts by thin film banding versus ameroid ring constrictor placement in dogs

\section{Matt Matiasovic, Ivan Doran, Guillaume Chanoit, Mickey Tivers}

University of Bristol Small Animal Hospital, Langford, UK

\section{OBJECTIVES}

The objective of this study was to retrospectively compare two cohorts of dogs surgically treated for single congenital extrahepatic portosystemic shunts by thin film banding or placement of an ameroid constrictor.

\section{METHODS}

Medical records of dogs with congenital extrahepatic portosystemic shunts treated by thin film banding or ameroid constrictor placement from 2009 to 2016 were reviewed for breed, age at surgery, duration of surgery, postsurgical complications, duration of hospitalisation, pre- and postoperative biochemical analysis, clinical outcome and requirement for repeat surgery. Data were reported with summary statistics.

\section{RESULTS}

Forty-nine dogs were treated with thin film banding and 23 were treated with ameroid constrictors based on surgeon preference. In the thin film banding group $10 \%$ of dogs died or were euthanased post-operatively compared to $4 \%$ in the ameroid group. Postoperative complications occurred in $24 \%$ of dogs treated by thin film banding and $22 \%$ of dogs treated with ameroid constrictors. Repeat surgery for further attenuation, due to persistent shunting was performed in $20 \%$ of dogs treated by thin film banding compared with $9 \%$ treated with ameroid constrictors.

\section{STATEMENT}

Our results suggest that ameroid constrictor placement is associated with lower postoperative mortality and a reduced need for second surgery compared to thin film banding. A relatively high rate of persistent shunting prompting additional surgery was seen in the dogs treated with thin film banding, suggesting that this method may not result in complete shunt attenuation in a significant proportion of animals, contrary to previously published findings.

\section{Monopolar burns as a complication of electrosurgery: a case series}

\section{Cesar Gomes², Heidi Radke ${ }^{1}$}

1 Queens Veterinary School Hospital, Cambridge, UK

2 Anderson and Abercromby Veterinary Referrals, Surrey, UK

\section{OBJECTIVES}

To describe cases of monopolar burns occurring as surgical complications, including their treatment and outcome, and to discuss strategies for their prevention.

\section{METHODS}

Cases where burns occurred as a result of monopolar diathermy use during surgery were reviewed (2011-2014). Cases with adequate medical history and record of follow-up procedures, treatments and outcome were included. 